\title{
Interactive comment on "Do alternative inventories converge on the spatiotemporal representation of spring ammonia emissions in France?" by Audrey Fortems-Cheiney et al.
}

\section{Anonymous Referee \#3}

Received and published: 1 July 2020

\section{GENERAL COMMENTS}

The manuscript submitted by Fortems-Cheiney et al. assesses different methods to determine $\mathrm{NH} 3$ emissions that are used in the scientific community. The authors compare three state-of-the art approaches for $\mathrm{NH} 3$ emission retrievals and discuss their spatial and temporal differences over a period in Spring 2011, which also includes the time of fertilizer application. While the analysis was performed for France, the discussed discrepancies between methods is also of high interest to researcher focusing on other regions. Due to the general high uncertainties in NH3 emission estimates, the presented study is of high relevance for improving chemical transport models. 
The manuscripts is well and clearly written and the analysis and interpretation of results is sound. Given the relevance of the topic for air quality, nitrogen deposition and atmospheric chemistry in general, I suggest approving the manuscript for publication in Atmospheric Chemistry and Physics after addressing the following comments:

\section{SPECIFIC COMMENTS}

L. 34-35: I suggest to clarify that the given values are the cumulative emissions from March to May. It is mentioned above, however, it would be good to restate here to not confuse it with an emissions flux.

L. 123: I assume the study period was from 1 March to 31 May? Still, the day should be included in the period description.

L. 217-219: Above it is stated that only morning overpass measurements are used. This may introduce a systematic bias to the NH3SAT emissions as emissions may be larger during midday due to higher temperatures or increased activity (e.g. mobile emissions). If I understand it correctly, the effect also depends on the TNO-GEN input data for CHIMERE, i.e. if monthly or a diurnal profile is applied to that. While I agree with the decision to only use morning overpass data, this potential bias should be explained and how it may impact the findings (e.g. could it explain why NH3SAT emissions are lower than those of CADASTRE-CIT in northeast, where fertilizer emission are high?).

L. 219-221: Could one also take only those periods where the IASI quality criteria are met and omit the other periods for the analysis instead of using the TNO-GEN emissions? How would that change the average correction factors presented in this study?

L. 246-247: From this paragraph alone it is not clear whether the manure field spreading is included the organic fertilizer application, which I would assume. If that is the case, you may add here "..., which is part of the organic fertilizer emissions" or similar. 
L. 314: In the Results \& Discussions section the authors describe the differences between the different approaches and also give here and there some suggestions why these differences occur. Still the discussions can be extended at the end of the section, e.g. by elaborating more on the uncertainties of each approach, leading to recommendations for future research directions and at which part each of the approaches should be improved (e.g. need for implementation of bi-directional exchange module in CHIMERE?). Although the paper is targeted on France, the findings are also insightful for users of $\mathrm{NH} 3$ emission estimates in other parts of the world. Therefore, relating the findings in the discussion also to inventories/approaches used in other regions would in my opinion make the manuscript stronger and attract more interest by a wider community.

L. 428: I suggest restating here in short the hypothesis from the introduction.

L. 405-408: Would market gardening be included in the TNO-GEN inventory? If not, an underestimation in that region would also apply to the TNO-GEN inventory.

\section{TECHNICAL COMMENTS}

Some words are accidentally merged, for example in lines $38,50,51,93,139,196$, 197, 207, 209, 210, 211, 272, 287, 291, 320, 371, 381, 400, 433, 466, 475 and 478.

The description and spelling of regions like "North-Eastern France" vs. "northeastern France" should be consistent throughout the manuscript.

L. 26: The sentence structure can be improved as it can be misleading what the actual methods are which the authors refer to. For example, I suggest inserting ":", "namely". . . or something similar after "emissions".

L. 292: I am not familiar with this terminology but there might be better terms than "desegregation" and "reagregation" in English.

L. 379: I suggest using "In bold are marked...". 
L. 401: A closing parenthesis is missing.

ACPD

Interactive comment on Atmos. Chem. Phys. Discuss., https://doi.org/10.5194/acp-2020-292, 2020.

Interactive

comment 